Objectives To examine gender and racial disparities in heart disease mortality related to metalworking fluid exposures and in the healthy worker survivor effect.

Method We examined ischaemic heart disease (IHD) mortality from 1941 to 1995 in a cohort of autoworkers with quantitative exposure to cumulative respirable particulate matter from waterbased metalworking fluids. Cox models were used to estimate the exposure-response to soluble and synthetic fluids separately in white men, black men, and white women. In separate analyses, we used g-estimation to adjust for the healthy worker survivor effect.

Results The risk of IHD was increased among black men (295 deaths) exposed to synthetic fluid with a hazard ratio (HR) of 3.47 (95\% CI: $1.52,7.92)$ in the highest cumulative exposure category. White women (119 deaths) had increased risk of IHD with increased soluble fluid (HR: $2.44(0.93,6.38)$ ) in the second to highest category. However, Cox models show no increased risk in white men (2246 deaths). In contrast, g-estimation results indicate that if white men had been always unexposed to soluble and synthetic fluid, then on average for each case, 2.99 and 2.77 years of life would have been saved, respectively.

Conclusions We found increased risk of IHD for black men and white women exposed to metalworking fluids using Cox regression. After adjusting for the healthy worker survivor effect, increased risk was observed for white men. The ability to leave work for health related reasons may be an option more available to white male workers.

\section{FARM EXPOSURES, ALLERGY SYMPTOMS AND RISK OF NON-HODGKIN LYMPHOMA IN THE AGRICULTURAL HEALTH STUDY}

${ }^{1} J$ onathan Hofmann, ${ }^{2}$ Jane Hoppin, ${ }^{1}$ Aaron Blair, ${ }^{1}$ Michael Alavanja, 'Laura Beane Freeman. ${ }^{1}$ National Cancer Institute, Bethesda, MD, USA; ${ }^{2}$ North Carolina State University, Raleigh, NC, USA

\subsection{6/oemed-2014-102362.34}

Objectives Exposure to allergens and microorganisms in the agricultural environment has been linked to altered immune response. Studies in the general population have reported reduced risks of non-Hodgkin lymphoma (NHL) among those with a history of atopic conditions, although results are inconsistent. To evaluate the allergy-NHL association in the context of farm exposures, we conducted an investigation in the Agricultural Health Study, a prospective cohort of farmers and spouses from North Carolina and Iowa.

Method Our study included 49656 farmers and spouses with crop and animal exposures and allergy symptoms reported at baseline (1993-1997). We identified 418 incident cases of NHL (including chronic lymphocytic leukaemia and multiple myeloma) during follow-up through 2010 in North Carolina and 2011 in Iowa. Hazard ratios (HR) and 95\% confidence intervals (CI) were calculated using multivariable-adjusted proportional hazards models.

Results At enrollment, over $80 \%$ of the study participants lived on farms growing grains or hay and $64 \%$ on farms raising livestock. Compared to individuals without allergy symptoms, those with symptoms had a reduced risk of NHL $(\mathrm{HR}=0.61,95 \%$ $\mathrm{CI}=0.50-0.74)$. We observed a slightly greater reduction in NHL risk among participants whose allergy symptoms worsened after working with grains and hay $(\mathrm{HR}=0.53,95 \% \mathrm{CI}=0.41-$ $0.69)$. The association between livestock and NHL was borderline significant overall $(\mathrm{HR}=0.82,95 \% \mathrm{CI}=0.66-1.01)$, and significant among those without allergy symptoms $(\mathrm{HR}=0.70$, $95 \% \mathrm{CI}=0.51-0.96)$.

Conclusions Our findings suggest that among individuals working and living on farms, allergy symptoms are associated with a reduced risk of developing NHL, and that risk may be influenced by particular farm characteristics.

\section{REDUCTION OF OCCUPATIONAL ACCIDENTS: EVIDENCE BASED PREVENTION AND THE PREVENTION INDEX (PI-TOP)}

${ }^{1}$ Frank Bochmann, ${ }^{2}$ Martin Arning, ${ }^{1} Y$ Y Sun, ${ }^{2}$ Jutta Boerger. ${ }^{1}$ IFA-Institute for Occupational Safety and Health of the German Social Accident Insurance, Sankt Augustin, Germany; ${ }^{2}$ German Social Accident Insurance Institution for the Woodworking and Metalworking Industries, Mainz, Germany

\subsection{6/oemed-2014-102362.35}

Objectives Prevention Index (PI-TOP) is a practical measurement tool which is currently used to monitor injury prevention activities at workplaces. This 12 -item scoring system has 3 subscales rating technical, organisational and personnel-related internal safety conditions of a company. The reliability and validity of this instrument were evaluated in a cross-sectional survey in the German metal industry during the time between December 2011 and May 2012.

Method The inter-rater-reliability of this instrument was examined by 2 trained supervisors of the German Social Accident Insurance Institution for the Woodworking and Metalworking Industries in 128 companies. The agreement of the double ratings was quantified by interclass correlation coefficient (ICC) and absolute agreement of the rating values. Construct validity of the score was examined by principle component factor analysis while content validity was evaluated by quantifying the association between PITOP-values and 5-years injury rates of 35000 companies. Poisson regression analysis was performed to assess the strength of the association adjusted for company size and related industrial sectors.

Results Our analysis indicate a moderate to good inter-rater-reliability (ICC $=0.46-0.75$ ) of PITOP-values with an absolute agreement between $72 \%$ and $81 \%$.

Factor analysis identified three component subscales which meet exactly the structural measure theory behind the score.

The Poisson regression analysis demonstrates that PITOP-values $\geq 7$ in the T-, $\mathrm{O}$ - and P-subscales are associated with a decrease of injury rates.

Conclusions Because this analysis indicates that PITOP is a valid and reliable instrument, it will be used to monitor safety conditions at workplaces in a longitudinal practical approach.

\section{PERSONAL ASSISTANCE - A PREREQUISITE TO WORK FOR PERSONS WITH SUBSTANTIAL AND PERMANENT DISABILITY?}

1,2leva Reine. 'Uppsala University, Uppsala, Sweden; '2Social Insurance Agency, Stockholm, Sweden

10.1136/oemed-2014-102362.36

Objectives In Sweden, people with a substantial and permanent disability have been entitled to personal assistance (PA) since the reform took effect in 1994. To qualify for government attendance allowance, a person must belong to one of the groups predefined in the Act Concerning Support and Service for Persons 